\title{
Anxiety and pain perception in patients undergoing mandibular autogenous block bone surgery
}

\author{
Luiz-Felipe-Silva Novy ${ }^{1}$, Evandro-Guimarães Aguiar ${ }^{2}$, José-Alcides-Almeida de Arruda ${ }^{1}$, Allyson-Nogueira \\ Moreira $^{3}$, Emerson-Gomes dos Santos ${ }^{4}$, Cláudia-Silami de Magalhães ${ }^{3}$, Amália Moreno ${ }^{2}$
}

${ }^{1}$ DDS, MSc, Department of Oral Surgery, Pathology and Clinical Dentistry, School of Dentistry, Universidade Federal de Minas Gerais, Belo Horizonte, Minas Gerais, Brazil

${ }^{2}$ DDS, PhD, Professor, Department of Oral Surgery, Pathology and Clinical Dentistry, School of Dentistry, Universidade Federal de Minas Gerais, Belo Horizonte, Minas Gerais, Brazil

${ }^{3}$ DDS, PhD, Professor, Department of Restorative Dentistry, School of Dentistry, Universidade Federal de Minas Gerais, Belo Horizonte, MG, Brazil

${ }^{4}$ BStat, PhD, Professor, Department of Business, Paulista School of Politics, Economics and Business, Universidade Federal de São Paulo, Osasco, SP, Brazil

\section{Correspondence:}

Department of Oral Surgery

Pathology and Clinical Dentistry

School of Dentistry

Universidade Federal de Minas Gerais

Belo Horizonte, Minas Gerais, Brazil

Av. Antônio Carlos, 6627. Pampulha

Belo Horizonte, MG, Brazil. CEP: 31.270-901

amalia_moreno@yahoo.com.br

Novy LFS, Aguiar EG, de Arruda JAA, Moreira AN, dos Santos EG, de Magalhães CS, Moreno A. Anxiety and pain perception in patients undergoing mandibular autogenous block bone surgery. J Clin Exp Dent. 2020;12(2):e130-8.

http://www.medicinaoral.com/odo/volumenes/v12i2/jcedv12i2p130.pdf

Received: 20/05/2019

Accepted: 25/11/2019

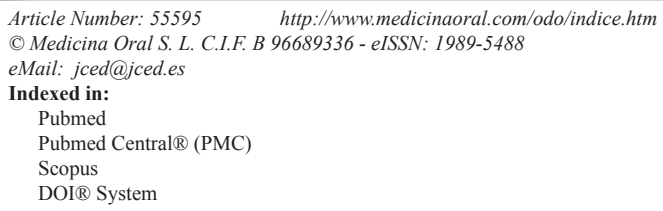

\begin{abstract}
Background: The aim of the present study was to investigate pain perception and anxiety within the context of surgery for the placement of mandibular block bone and to evaluate the causality effect between theses variables. Material and Methods: A total of 13 patients were recruited for the study and were submitted to mandibular autogenous block bone surgery. Demographic data were collected and the anxiety level was determined using the State-Trait Anxiety Inventory (STAI). The STAI was administered on the day of surgery and on the 14th postoperative day. Pain was determined using the visual analogue scale (VAS) and limitation of daily activities and postoperative symptoms were also reported. Data were analyzed using parametric tests $(\alpha=0.05)$ and cross-lagged analysis was performed to verify a causality effect.

Results: Few patients reported interference with daily activities or the presence of postoperative symptoms. A significant association of bad breath/taste with STAI-State was detected on the 14th postoperative day. No evidence of causality between STAI and VAS was detected.

Conclusions: The patient's self-evaluation indicates that the pain and anxiety level felt during treatment was not directly associated with the clinical aspects of the surgical procedure or with postoperative activities/symptoms limitations.
\end{abstract}

Key words: Anxiety pain, questionnaires, autogenous bone block, treatment, outcomes. 


\section{Introduction}

Oral surgical procedures are common for individuals requiring rehabilitation with dental implants or after bone grafts surgeries (1). Autogenous intraoral bone grafts have been widely employed to increase the bone volume of the alveolar crest due to their reliability and predictability (2). The use of autogenous block grafts for vertical and horizontal bone augmentation has been reported, with a low rate of complications and satisfactory bone regeneration at the donor site $(3,4)$.

These procedures are known to involve mild to moderate pain, causing stress and short-term limitations of daily activities, especially during the first postoperative days $(5,6)$. In addition, previous studies have demonstrated an impact of patient anxiety on factors related to dental implants surgery $(7,8)$. The control of anxiety levels in patients submitted to oral and maxillofacial surgeries may be directly related to the subjective control of pain and its impact on daily activities, with a consequent reduction of morbidity in the operated region $(9,10)$.

Dental anxiety has not been clearly defined in the literature, ranging from the mildest feeling of apprehension before a clinical procedure to the strongest panic attack with impairment of dental treatment (11). Gaudry et al. (12) described the difference between anxiety as a transitory state in the face of threatening stimuli and as a personality trait. State anxiety is based on subjective and conscious feelings of apprehension and tension accompanied by, or associated with, activation of the autonomic nervous system. In contrast, trait anxiety implies a motive or an acquired behavioral disposition that predisposes an individual to perceive as threatening circumstances that are not objectively dangerous and to react with state anxiety of disproportionate intensity related to the magnitude of danger (12).

The emotional status of an individual who undergoes dental surgery may generate or worsen a degree of anxiety that will have some type of impact on the treatment effectiveness. Although the effect of anxiety on patient pain after dental implant or extraction procedures has been investigated $(6,13)$ to our knowledge, no reports on patients submitted to bone graft surgeries are currently available. Intraoral bone grafting may be affected by various factors such as duration of surgery, trans- and postoperative intercurrences, duration of pain, and recovery, which contribute to a state of stress. Thus, the aim of the present study was to evaluate the pain perception and anxiety related to autogenous mandibular block bone surgery using visual analog scales (VAS), the State-Trait Anxiety Inventory (STAI), and information about treatment and follow-up data. Also, we evaluated the causality effect of anxiety on pain perception during the treatment period.

\section{Material and Methods}

-Study design and ethical issues

A prospective cohort study was conducted on individuals seen at the Service of Oral Surgery and Implantology of the School of Dentistry, Federal University of Minas Gerais (MG, Brazil) between July and December 2017. The study was approved by the local Ethics Committee (Approval No. 67497617.7.0000.5149). The participants gave written informed consent in agreement with the Declaration of Helsinki.

-Patients

A total of 13 individuals were included in the study according to the criteria: adult or elderly needing a thick bone graft in the posterior mandible as determined by visual examination and palpation and confirmed by cone beam computed tomography (CBCT); $3.0 \mathrm{~mm}$ to 8.0 $\mathrm{mm}$ bone height remaining above the mandibular canal roof; valley-shaped bone defect with a $2.0 \mathrm{~mm}$ minimum depth in relation to the bone crest, with indication of oral rehabilitation with bone integrable implants; and cognitive ability to understand and answer the questionnaires. Exclusion criteria were as follows: general contraindications of surgical treatment, being under treatment or taking medications that interfere with tissue repair, smokers and alcoholics, presence of active infectious process in the region to be operated, and a history of implant or graft installation in the mandible.

Gender, age, household income, reason for the dentist's appointment of the subjects were recorded. Intraoral physical examination was performed, and preoperative laboratory tests and CBCT images were evaluated. The responses to two self-applicable questionnaires were collected.

-Surgical procedures

All surgical procedures were performed by the same senior maxillofacial surgeon (E.G.A.). Two calibrated examiners (L.S.N. and C.S.M.) conducted the clinical examination and collected the VAS and questionnaire data. The autogenous bone graft was obtained from the external oblique ridge according to Khoury et al. (4), with some modifications. The following preoperative medication was used: amoxicillin $(875 \mathrm{mg}$, administered at least 1 hour prior to surgery), tenoxicam ( $20 \mathrm{mg}$, at least 1 hour prior to surgery), dexamethasone (two $4 \mathrm{mg}$ capsules, at least 12 hours prior to surgery, and $8 \mathrm{mg}$ at least 2 hours prior to surgery), and paracetamol (750 $\mathrm{mg}$, at least 1 hour prior to surgery).

Perforations were punched in the anesthetized donor area with an NR699 drill (Solidor Ind., SP, Brazil) in parallel to the external oblique area, reaching the full thickness of cortical bone. Mesial and distal vertical osteotomy lines were performed under constant irrigation with saline $\left(5^{\circ} \mathrm{C}\right)$, determining the length of the graft. The vertical osteotomies were then joined with a hori- 
zontal osteotomy at the basal level using a diamond disk ( $5 \mathrm{~mm}$, NeoBiotech, CA, USA). Particulated bone from a region adjacent to the donor bed was obtained with the aid of an ACM drill (NeoBiotech, CA, USA). The bone block was carefully removed with a chisel and divided longitudinally in the middle into thin parts with a diamond disk (Fig. 1). The edges were rounded and the blocks were stabilized in the recipient regions at a distance from the bone bed and maintained with titanium screws (1.5 mm, NeoBiotech, CA, USA) (Fig. 1) manually inserted using a screwdriver key (square active tip, PecLab Ind., MG, Brazil). The first block was fixed in the occlusal region resting on the bone crest of the adjacent tooth. The space below the first block was filled with particulate bone and the second block was then installed on the vestibular surface. instructed to perform mouthwashes with $5 \mathrm{~mL} 0.12 \%$ chlorhexidine gluconate for 1-minute half an hour after oral hygiene, three times a day for 7 days.

All participants were evaluated seven days after surgery and the sutures were removed after 10 days, when clinical reevaluation was performed. The patients were instructed not to apply a direct load to the reconstructed region with the use of dental prostheses throughout the phase of bone regeneration. After the 14th postoperative day the patients were asked whether they experienced interference with their daily activities such as chewing, full mouth opening, speaking, sleeping, job, social life, or favorite activities. The patients were also asked to report postoperative symptoms such as the presence of facial swelling, nausea, and the perception of bad breath/ taste.

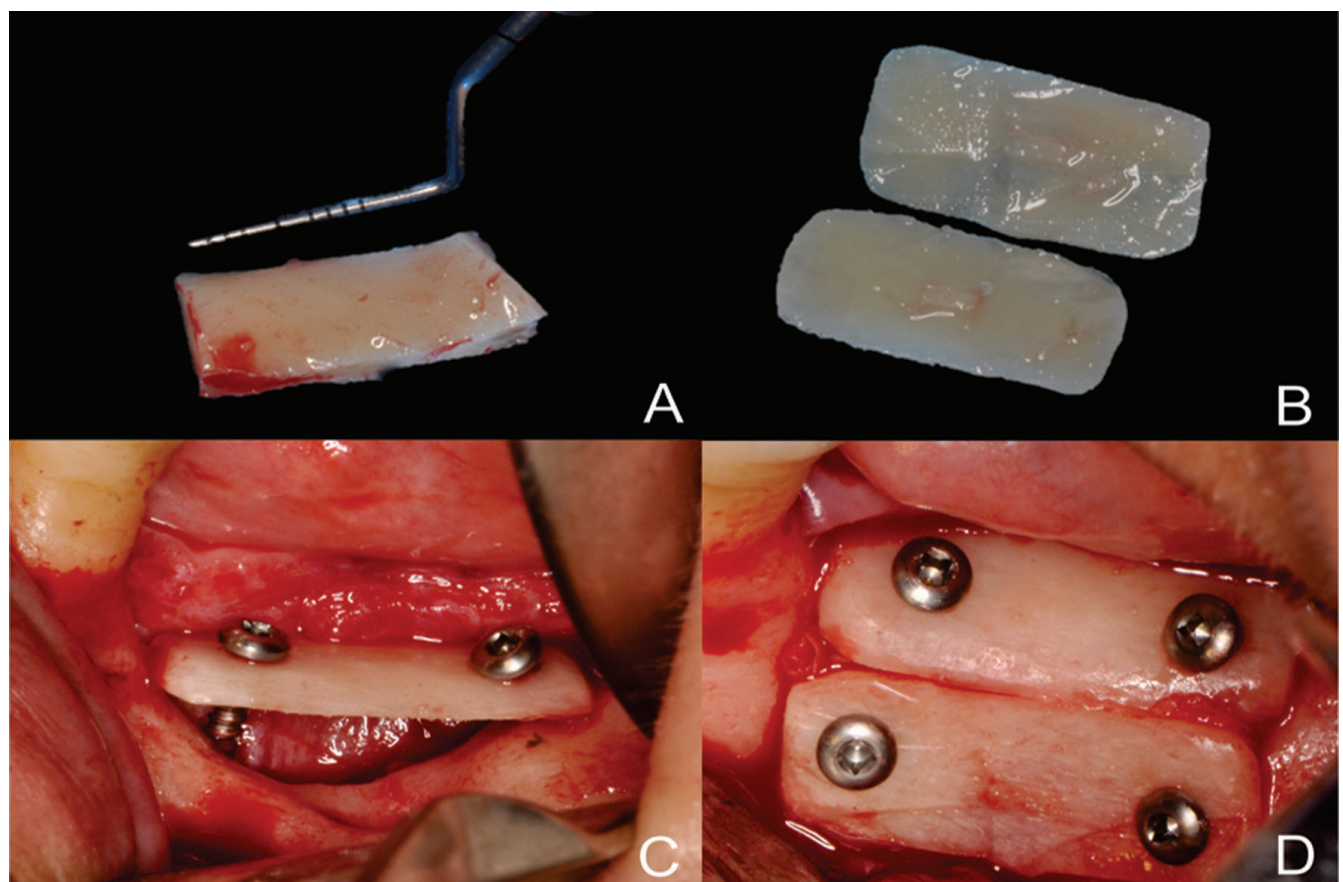

Fig. 1: (A) Harvesting two bone blocks from the mandible, (B) splitting them into two thinner blocks, and (C,D) placement of bone blocks in occlusal and vestibular bone cortices.

The surgical wounds in the recipient and in the donor areas were then closed with simple non-restorable sutures (Nylon 6-0, Ethicon, NJ, USA). The number of anesthetic tubes used, the surgical time and transoperatory intercurrences were recorded.

-Follow-up and daily activities/postoperative symptoms Medication to be continued postoperatively with amoxicillin ( $875 \mathrm{mg}$ twice a day for 7 days), tenoxicam (20 $\mathrm{mg}$ for 5 days), dexamethasone ( $8 \mathrm{mg}$ twice a day for 5 days), and paracetamol (750 $\mathrm{mg}$ four times/day for two days). After the third postoperative day, the patients were

\section{-STAI}

For the assessment of anxiety, the patients responded to two self-assessments questionnaires $(14,15)$ : STAI-State (STAI-S), and STAI-Trace (STAI-T), seven days before surgery (phase 1), immediately (30 minutes) before surgery (phase 2) and 14 days after surgery (phase 3). The state anxiety scale consists of 20 statements that assess how the respondent feels "at this time". The trait anxiety scale consists of 20 statements that assess how the respondent "generally feels". Both scales evaluate feelings of apprehension, tension, nervousness and wo- 
rry. The patients expressed each statement using a 0 to 4 Likert-type scale.

-VAS

Each patient performed the VAS test, recording the perception of pain on a scale from 0 to 10 , with 0 corresponding to "no pain" and 10 to "the worst pain ever felt". The test was performed 30 minutes after bone graft surgery (phase 2), and again 14 days after surgery (phase 3).

-Statistical analysis

Demographic data and the reasons for seeking dental treatment were submitted to descriptive analyses using the Statistical Package for the Social Sciences software (SPSS), version 22.0 (IBM Inc., New Armonk, NJ, USA). The sum of STAI scores for all questions for each patient was calculated. Scores of 20-40 represented a low level of anxiety and scores of 41-80 represented an medium to high level of anxiety. The responses to the VAS were dichotomized as "without pain" for zero scores and "with pain" for scores from 1 to 10 . The hypothesis of association between clinical variables and STAI was tested at phase 2; between daily activities/ postoperative symptoms and VAS at phase 3 , and STAI at phase 3. Fisher's exact test of independence $(\alpha=0.05)$ was used to verify the hypothesis of association as the sample size is small.

STAI sums of scores were compared at 3 time points (phases 1,2 and 3) using the Friedman test. A post-hoc test ( $\alpha=0.05$ ) was employed using the PMCMR package of the R software (R Core Team, Vienna, Austria). The hypothesis of no association between the patients' STAI and VAS were tested by cross-lagged analysis using the COCOR package (16) of the R software. Thus, three types of correlations were performed: autocorrelations (between two measures of a variable at different times), synchronous correlations (between different variables at the same time) and cross-lagged correlations (between different variables at different times). The difference between cross-lagged analyses was determined by the modified Pearson-Filon ZPF test.

\section{Results}

The sociodemographic data of the individuals are shown in Table 1. The sample consisted of nine women and four men (mean age: 53.4 years). Approximately 50\% of the individuals had household incomes lower than 3 times the Brazilian minimum wage, and the other 50\% had incomes higher than this level. The main reason for the dental appointment was the recovery of missing teeth $(69.2 \%)$.

No significant association between clinical variables and STAI ( $p>0.05)$ was observed at phase 2 (Table 2$)$. Some factors such as duration of surgery $(p=0.078)$, complications during surgery $(p=0.070)$ and number of anesthetic cartridges used $(p=0.098)$ may have been influenced statistically by anxiety considering an interval estimate with a $90 \%$ confidence level (Table 2). Considering some influence of surgery duration on anxiety five (71.4\%) subjects whose surgery lasted more than 120 minutes showed medium to hight anxiety on STAI-S at phase 2 , in contrast to one $(16.7 \%)$ patient whose surgery lasted less than 120 minutes $(p=0.078)$. Only three patients showed complications during surgery, also showing average to high anxiety on STAI-S at phase 2 $(p=0.078)$ (Table 2).

In addition, regarding daily activities/postoperative symptoms, an association was detected between bad breath/taste and STAI-S at phase $3(p=0.014)$. Three of the four $(30.8 \%)$ patients who reported bad breath/taste $(75.0 \%)$ showed medium to high anxiety on STAI-S at phase $3(p=0.014)$. There was no significant association between STAI at phase 2 and other variables $(p>0.05)$ (Table 3).

Regarding association between activities/symptoms and VAS at phase 2 , four (30.8\%) patients complained of interference with their favorite activities and two (50\%)

Table 1: Sociodemographic data of participants undergoing bone surgery.

\begin{tabular}{|l|c|}
\hline Variables & $\mathbf{n}(\mathbf{\%})$ \\
\hline Gender & \\
\hline Female & $9(69.2)$ \\
\hline Male & $4(30.8)$ \\
\hline Age, years (range; mean \pm SD) & $25-68 ; 53.4 \pm 11.7$ \\
\hline Household income & $6(46.2)$ \\
\hline$<3$ times the Brazilian minimum wage & $7(53.8)$ \\
\hline Between $\geq 3$ and 5 times the Brazilian minimum wage & \\
\hline Reason for the dentist's appointment & $9(69.2)$ \\
\hline Absence of posterior teeth & $4(30.8)$ \\
\hline Performing implants & \\
\hline
\end{tabular}


Table 2: Association between State-Trait Anxiety Inventory (STAI) scores and clinical variables.

\begin{tabular}{|c|c|c|c|c|c|c|c|c|c|c|c|c|c|c|}
\hline \multirow[t]{3}{*}{ Clinical variables } & \multicolumn{7}{|c|}{ STAI-S phase 2} & \multicolumn{7}{|c|}{ STAI-T phase 2} \\
\hline & \multicolumn{2}{|c|}{ Low } & \multicolumn{2}{|c|}{$\begin{array}{l}\text { medium to } \\
\text { high }\end{array}$} & \multicolumn{2}{|c|}{ Total } & \multirow[t]{2}{*}{$p$-value ${ }^{\dagger}$} & \multicolumn{2}{|c|}{ Low } & \multicolumn{2}{|c|}{$\begin{array}{l}\text { Medium } \\
\text { to high }\end{array}$} & \multicolumn{2}{|c|}{ Total } & \multirow[t]{2}{*}{$p$-valu ${ }^{\text {e }}$} \\
\hline & $\mathbf{n}$ & $\%$ & $\mathbf{n}$ & $\%$ & $\mathbf{n}$ & $\%$ & & $\mathbf{n}$ & $\%$ & $\mathbf{n}$ & $\%$ & $\mathbf{n}$ & $\%$ & \\
\hline \multicolumn{15}{|l|}{ Medical care } \\
\hline No & 6 & 60 & 4 & 40 & 10 & 76.9 & \multirow[t]{2}{*}{$0.437^{\mathrm{ns}}$} & 7 & 70.0 & 3 & 30.0 & 10 & 76.9 & \multirow[t]{2}{*}{$0.315^{\mathrm{ns}}$} \\
\hline Yes & 1 & 33.3 & 2 & 66.7 & 3 & 23.1 & & 1 & 33.3 & 2 & 66.7 & 3 & 23.1 & \\
\hline \multicolumn{15}{|c|}{ Continuous-use medicine } \\
\hline No & 4 & 66.7 & 2 & 33.3 & 6 & 46.2 & \multirow[t]{2}{*}{$0.383^{\text {ns }}$} & 5 & 83.3 & 1 & 16.7 & 6 & 46.2 & \multirow[t]{2}{*}{$0.179^{\text {ns }}$} \\
\hline Yes & 3 & 42.9 & 4 & 57.1 & 7 & 53.8 & & 3 & 42.9 & 4 & 57.1 & 7 & 53.8 & \\
\hline \multicolumn{15}{|c|}{ Number of anesthetic cartridges } \\
\hline$\leq 6$ & 2 & 50.0 & 2 & 50.0 & 4 & 30.8 & \multirow[t]{2}{*}{$0.657^{\text {ns }}$} & 4 & 100 & 0 & 0 & 4 & 30.8 & \multirow[t]{2}{*}{$0.098^{*}$} \\
\hline$>6$ & 5 & 55.6 & 4 & 44.4 & 9 & 69.2 & & 4 & 44.4 & 5 & 55.6 & 9 & 69.2 & \\
\hline \multicolumn{15}{|c|}{ Duration of surgery (minutes) } \\
\hline$\leq 120$ & 5 & 83.3 & 1 & 16.7 & 6 & 46.2 & \multirow[t]{2}{*}{$0.078^{*}$} & 4 & 66.7 & 2 & 33.3 & 6 & 46.2 & \multirow[t]{2}{*}{$0.587^{\text {ns }}$} \\
\hline$>120$ & 2 & 28.6 & 5 & 71.4 & 7 & 53.8 & & 4 & 57.1 & 3 & 42.9 & 7 & 53.8 & \\
\hline \multicolumn{15}{|c|}{ Complications during surgery } \\
\hline No & 7 & 70.0 & 3 & 30.0 & 10 & 76.9 & \multirow[t]{2}{*}{$0.070^{*}$} & 7 & 70.0 & 3 & 30.0 & 10 & 76.9 & \multirow[t]{2}{*}{$0.315^{\mathrm{ns}}$} \\
\hline Yes & 0 & 0 & 3 & 100.0 & 3 & 23.1 & & 1 & 33.3 & 2 & 66.7 & 3 & 23.1 & \\
\hline \multicolumn{15}{|c|}{ Complication after surgery } \\
\hline No & 2 & 33.3 & 4 & 66.7 & 6 & 46.2 & \multirow[t]{2}{*}{$0.209^{\text {ns }}$} & 3 & 50.0 & 3 & 50.0 & 6 & 46.2 & \multirow[t]{2}{*}{$0.413^{\text {ns }}$} \\
\hline Yes & 5 & 71.4 & 2 & 28.6 & 7 & 53.8 & & 5 & 71.4 & 2 & 28.6 & 7 & 53.8 & \\
\hline Total & 7 & 53.8 & 6 & 46.2 & 13 & 100.0 & & 8 & 61.5 & 5 & 38.5 & 13 & 100.0 & \\
\hline
\end{tabular}

†Fisher test

ns: $p>0.1$

$* p<0.1$

reported some level of pain (Table 4). Also, there was no significant difference for STAI between the three phases of this study ( $p>0.05$, Friedman test) (Fig. 2). Regarding VAS, six patients reported pain at phase 2 compared with two patients at phase 3 , with a statistically significant difference between phases 2 and 3 ( $p=0.014$, Wilcoxon test).

The autocorrelations in the STAI responses between phases 2 and 3 were significant ( $\mathrm{r}=0.76, p=0.003$ to STAI-S; $\mathrm{r}=0.79, p=0.001$ to STAI-T). There were no significant differences in synchronous correlations at phases 2 or 3 between STAI responses and VAS. Cross-lagged analysis showed significant causality only between VAS at responses phase 2 and STAI-T responses at phase 3 $(\mathrm{r}=0.57 ; p=0.044)$. In general, cross-lagged analysis of the data did not show significant causality between STAI and VAS responses (ZPF test, $p>0.05$ ) (Fig. 3).

\section{Discussion}

Marked bone loss in the edentulous region of the posterior mandible requires surgical procedures in order to obtain vertical and horizontal bone gain before treatment with dental implants $(2,17)$. Since surgical procedures are considered to be negative by involving pain, the present study revealed that $69.2 \%$ of the patients studied sought oral rehabilitation in order to restore the lost posterior teeth, whereas a minority of the sample seemed to already have been informed about surgery for dental implants and to be willing to undergo such treatment. Moreover, the emotional status of medium to high anxiety of same patients at phase 2 was associated with some factors inherent to the procedure such as surgical time, complications and number of anesthetic cartridges during the operation. This fact may be related to evidence that more anxious patients are usually restless and interfere with the progression of the surgical procedure $(8,18)$.

Interference with routine activities (e.g., working or studying) has been reported during the first three postoperative days, followed by reduced pain along the first postoperative week (5). These findings agree with the results of the present study, since most patients submitted to bone graft surgery reported pain during the immediate postoperative period, with a significant diffe- 
Table 3: Association between State-Trait Anxiety Inventory (STAI) scores and daily activities/postoperative symptoms.

\begin{tabular}{|c|c|c|c|c|c|c|c|c|c|c|c|c|c|c|}
\hline \multirow{3}{*}{$\begin{array}{l}\text { Activities/ } \\
\text { postoperative } \\
\text { symptoms }\end{array}$} & \multicolumn{7}{|c|}{ STAI-S phase 3} & \multicolumn{7}{|c|}{ STAI-T phase 3} \\
\hline & \multicolumn{2}{|c|}{ Low } & \multicolumn{2}{|c|}{$\begin{array}{l}\text { Medium } \\
\text { to high }\end{array}$} & \multicolumn{2}{|c|}{ Total } & \multirow[t]{2}{*}{$p$-value ${ }^{\dagger}$} & \multicolumn{2}{|c|}{ Low } & \multicolumn{2}{|c|}{$\begin{array}{l}\text { Medium } \\
\text { to high }\end{array}$} & \multicolumn{2}{|c|}{ Total } & \multirow[t]{2}{*}{$p$-value ${ }^{\dagger}$} \\
\hline & $n$ & $\%^{a}$ & $n$ & $\%^{a}$ & $n$ & $\%^{b}$ & & $n$ & $\%^{a}$ & $n$ & $\%^{\mathrm{a}}$ & $n$ & $\%^{\mathrm{b}}$ & \\
\hline \multicolumn{15}{|l|}{ Chewing } \\
\hline No & 4 & 66.7 & 2 & 33.3 & 6 & 46.2 & \multirow[t]{2}{*}{$0.437^{\mathrm{ns}}$} & 4 & 66.7 & 2 & 33.3 & 6 & 46.2 & \multirow[t]{2}{*}{$0.437^{\mathrm{ns}}$} \\
\hline Yes & 6 & 85.7 & 1 & 14.3 & 7 & 53.8 & & 6 & 85.7 & 1 & 14.3 & 7 & 53.8 & \\
\hline \multicolumn{15}{|l|}{ Mouth opening } \\
\hline No & 7 & 77.8 & 2 & 22.2 & 9 & 69.2 & \multirow[t]{2}{*}{$0.706^{\text {ns }}$} & 7 & 77.8 & 2 & 22.2 & 9 & 69.2 & \multirow[t]{2}{*}{$0.706^{\text {ns }}$} \\
\hline Yes & 3 & 75.0 & 1 & 25.0 & 4 & 30.8 & & 3 & 75.0 & 1 & 25.0 & 4 & 30.8 & \\
\hline \multicolumn{15}{|l|}{ Speaking } \\
\hline No & 6 & 75.0 & 2 & 25.0 & 8 & 61.5 & \multirow[t]{2}{*}{$0.685^{\text {ns }}$} & 6 & 75.0 & 2 & 25.0 & 8 & 61.5 & \multirow[t]{2}{*}{$0.685^{\text {ns }}$} \\
\hline Yes & 4 & 80.0 & 1 & 20.0 & 5 & 38.5 & & 4 & 80.0 & 1 & 20.0 & 5 & 38.5 & \\
\hline \multicolumn{15}{|l|}{ Sleep } \\
\hline No & 9 & 90.0 & 1 & 10.0 & 10 & 76.9 & \multirow[t]{2}{*}{$0.108^{\text {ns }}$} & 8 & 80.0 & 2 & 20.0 & 10 & 76.9 & \multirow[t]{2}{*}{$0.580^{\mathrm{ns}}$} \\
\hline Yes & 1 & 66.7 & 2 & 33.3 & 3 & 23.1 & & 2 & 66.7 & 1 & 33.3 & 3 & 23.1 & \\
\hline \multicolumn{15}{|l|}{ Job } \\
\hline No & 8 & 72.7 & 3 & 27.3 & 11 & 84.6 & \multirow[t]{2}{*}{$0.577^{\mathrm{ns}}$} & 8 & 72.7 & 3 & 27.3 & 11 & 84.6 & $0.577^{\mathrm{ns}}$ \\
\hline Yes & 2 & 100 & 0 & 0 & 2 & 15.4 & & 2 & 100.0 & 0 & 0 & 2 & 15.4 & \\
\hline Social life & & & & & & & & & & & & & & \\
\hline No & 7 & 77.8 & 2 & 22.2 & 9 & 69.2 & $0.706^{\mathrm{ns}}$ & 7 & 77.8 & 2 & 22.2 & 9 & 69.2 & $0.706^{\text {ns }}$ \\
\hline Yes & 3 & 75.0 & 1 & 25.0 & 4 & 30.8 & & 3 & 75.0 & 1 & 25.0 & 4 & 30.8 & \\
\hline $\begin{array}{l}\text { Favorite } \\
\text { activities }\end{array}$ & & & & & & & & & & & & & & \\
\hline No & 6 & 66.7 & 3 & 33.3 & 9 & 69.2 & $0.294^{\mathrm{ns}}$ & 6 & 100.0 & 3 & 0 & 9 & 69.2 & $0.294^{\mathrm{ns}}$ \\
\hline Yes & 4 & 100.0 & 0 & 0 & 4 & 30.8 & & 4 & 100.0 & 0 & 0 & 4 & 30.8 & \\
\hline Facial swelling & & & & & & & & & & & & & & \\
\hline No & 7 & 77.8 & 2 & 22.2 & 9 & 69.2 & $0.706^{\mathrm{ns}}$ & 7 & 77.8 & 2 & 22.2 & 9 & 69.2 & $0.706^{\mathrm{ns}}$ \\
\hline Yes & 3 & 75.0 & 1 & 25.0 & 4 & 30.8 & & 3 & 75.0 & 1 & 25.0 & 4 & 30.8 & \\
\hline Nausea & & & & & & & & & & & & & & \\
\hline No & 8 & 72.7 & 3 & 27.3 & 11 & 84.6 & $0.577^{\mathrm{ns}}$ & 8 & 72.7 & 3 & 27.3 & 11 & 84.6 & $0.577^{\mathrm{ns}}$ \\
\hline Yes & 2 & 100.0 & 0 & 0 & 2 & 15.4 & & 2 & 100.0 & 0 & 0 & 2 & 15.4 & \\
\hline Bad breath/taste & & & & & & & & & & & & & & \\
\hline No & 9 & 100.0 & 0 & 0 & 9 & 69.2 & $0.014^{*}$ & 8 & 88.9 & 1 & 11.1 & 9 & 69.2 & $0.203^{\text {ns }}$ \\
\hline Yes & 1 & 25.0 & 3 & 75.0 & 4 & 30.8 & & 2 & 50.0 & 2 & 50.0 & 4 & 30.8 & \\
\hline Total & 10 & 76.9 & 3 & 23.1 & 13 & 100.0 & & 11 & 84.6 & 2 & 15.4 & 13 & 100.0 & \\
\hline
\end{tabular}

$\dagger$ Fisher test

ns: $p>0.05$

$* p<0.05$ 
Table 4: Association between visual analog scales (VAS) and daily activities/postoperative symptoms.

\begin{tabular}{|c|c|c|c|c|c|c|c|}
\hline \multirow{3}{*}{$\begin{array}{l}\text { Activities/postoperative } \\
\text { symptoms }\end{array}$} & \multicolumn{7}{|c|}{ VAS phase 3} \\
\hline & \multicolumn{2}{|c|}{ No } & \multicolumn{2}{|c|}{ Yes } & \multicolumn{2}{|c|}{ Total } & \multirow[b]{2}{*}{$p$-value ${ }^{\dagger}$} \\
\hline & $\mathbf{n}$ & $\%^{a}$ & $\mathbf{n}$ & $\%^{a}$ & n & $\%^{\mathbf{b}}$ & \\
\hline \multicolumn{8}{|l|}{ Chewing } \\
\hline No & 6 & 100.0 & 0 & 0 & 6 & 46.2 & \multirow[t]{2}{*}{$0.269^{\mathrm{ns}}$} \\
\hline Yes & 5 & 71.4 & 2 & 28.6 & 7 & 53.8 & \\
\hline \multicolumn{8}{|l|}{ Mouth opening } \\
\hline No & 8 & 88.9 & 1 & 11.1 & 9 & 69.2 & \multirow[t]{2}{*}{$0.538^{\mathrm{ns}}$} \\
\hline Yes & 3 & 75.0 & 1 & 25.0 & 4 & 30.8 & \\
\hline \multicolumn{8}{|l|}{ Speaking } \\
\hline No & 7 & 87.5 & 1 & 12.5 & 8 & 61.5 & \multirow[t]{2}{*}{$0.641^{\mathrm{ns}}$} \\
\hline Yes & 4 & 80.0 & 1 & 20.0 & 5 & 38.5 & \\
\hline \multicolumn{8}{|l|}{ Sleep } \\
\hline No & 9 & 90.0 & 1 & 10.0 & 10 & 76.9 & \multirow[t]{2}{*}{$0.423^{\mathrm{ns}}$} \\
\hline Yes & 2 & 66.7 & 1 & 33.3 & 3 & 23.1 & \\
\hline \multicolumn{8}{|l|}{ Job } \\
\hline No & 10 & 90.9 & 1 & 9.1 & 11 & 84.6 & \multirow[t]{2}{*}{$0.295^{\text {ns }}$} \\
\hline Yes & 1 & 50.0 & 1 & 50.0 & 2 & 15.4 & \\
\hline \multicolumn{8}{|l|}{ Social life } \\
\hline No & 8 & 88.9 & 1 & 11.1 & 9 & 69.2 & $0.538^{\mathrm{ns}}$ \\
\hline Yes & 3 & 75.0 & 1 & 25.0 & 4 & 30.8 & \\
\hline \multicolumn{8}{|l|}{ Favorite activities } \\
\hline No & 9 & 100.0 & 0 & 0 & 9 & 69.2 & \multirow[t]{2}{*}{$0.077^{*}$} \\
\hline Yes & 2 & 50.0 & 2 & 50.0 & 4 & 30.8 & \\
\hline \multicolumn{8}{|l|}{ Facial swelling } \\
\hline No & 8 & 88.9 & 1 & 11.1 & 9 & 69.2 & \multirow[t]{2}{*}{$0.538^{\mathrm{ns}}$} \\
\hline Yes & 3 & 75.0 & 1 & 25.0 & 4 & 30.8 & \\
\hline \multicolumn{8}{|l|}{ Nausea } \\
\hline No & 9 & 81.8 & 2 & 18.2 & 11 & 84.6 & \multirow[t]{2}{*}{$0.705^{\text {ns }}$} \\
\hline Yes & 2 & 100.0 & 0 & 0 & 2 & 15.4 & \\
\hline \multicolumn{8}{|l|}{ Bad breath/taste } \\
\hline No & 8 & 88.9 & 1 & 11.1 & 9 & 69.2 & \multirow[t]{2}{*}{$0.538^{\mathrm{ns}}$} \\
\hline Yes & 3 & 75.0 & 1 & 25.0 & 4 & 30.8 & \\
\hline Total & 11 & 84.6 & 2 & 15.4 & 13 & 100.0 & \\
\hline
\end{tabular}

$\dagger$ Fisher test

ns: $p>0.1$

$* p<0.1$ 

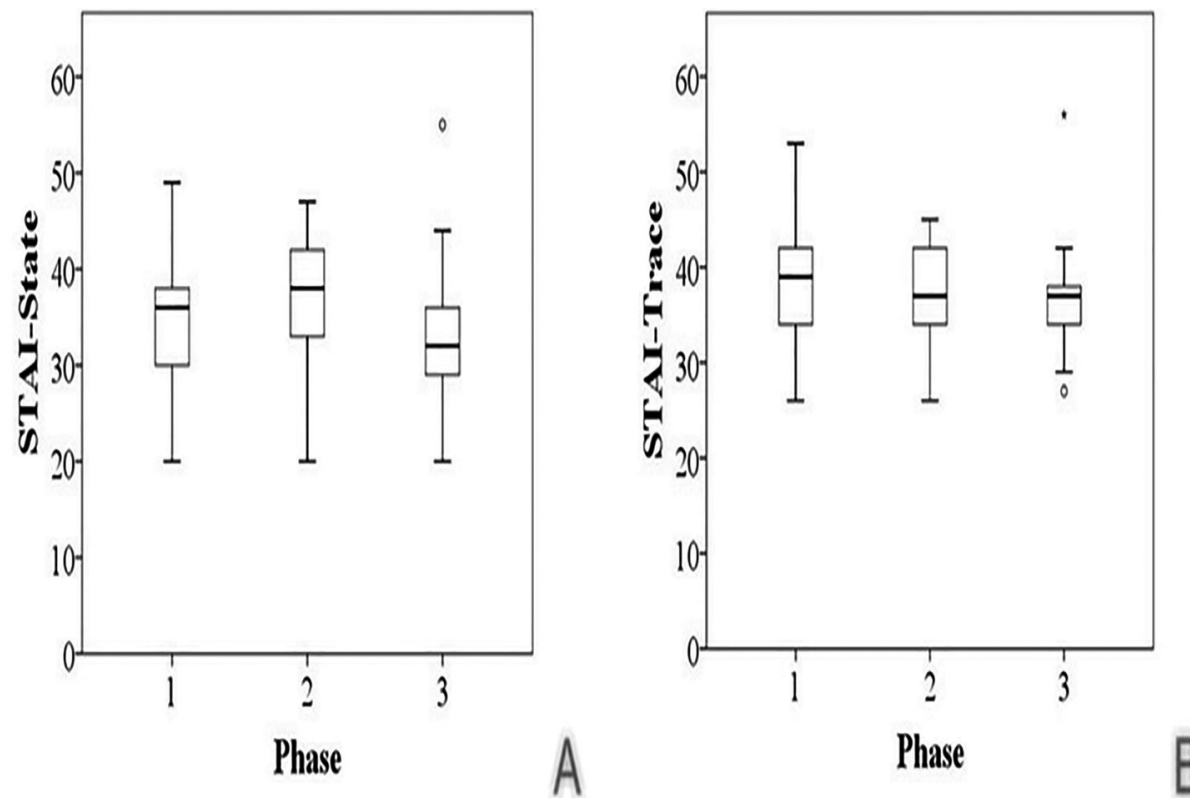

Fig. 2: Box Plot of STAI-S (A) and STAI-T (B) at different phases of the study.


Fig. 3: Cross-lagged analysis for STAI-S and VAS (A) and STAI-T and VAS (B).

rence compared to 14 days after surgery. However, this fact may be probably due to the comfortable condition provided by the surgical procedure. Also, the fact that most limitations of daily activities and the postoperative symptoms did not show an association with anxiety is due to the good oral and systemic conditions provided by graft surgery. Similar studies have reported that patients experience pain of mild to moderate intensity in surgeries for dental extraction or for surgical implants, usually showing a good recovery, including monitoring for future procedures $(5,8,19)$. However, bad breath was observed in association with STAI-S. Our findings agree with the study of Muglali et al. (19), who reported that some underestimated factors related to oral health may be associated with the presence or absence of a reduction of patient anxiety before and after oral surgery.

Herein, there was no significant difference during the phases of evaluation of anxiety, a fact that supports the permanence of the emotional status and the expectation about the treatment received. Although cross-lagged analysis did not show a statistically significant difference in causality, it can be seen that the pain occurring in phase 2 may have influenced the anxiety during phase 3 in the STAI-T. The factors that may be involved in anxiety are the age of the subjects, their psychological conditions, previous medical experiences, different degrees of sensitivity to, or tolerance of, pain, influence of the family or the peer group regarding the potentiation or minimization of the personality status, as well as the reaction to the place of care (20). Previous studies have investigated the theme of origin of dental anxiety, its main causes and consequences related to the fear previously shown by the patient $(21,22)$. In addition, dental fear and anxiety may have generalized psychosocial consequences, explaining the permanence or increase of anxiety during treatment (22).

A positive association between anxiety and the perception of pain in surgical dental procedures was reported 
$(6,23)$. Moreover, the technical procedure and the surgical skill of the operator are important factors for the success of intraoral bone graft surgery, as the patient emotional control becomes a requisite for surgical success (23). Thus, it is important for the professionals to understand that a painful experience can influence anxiety and that the anxiety state is related to changes in the execution of future treatments.

The limitations of the present study are due to the aspects inherent to the design of a prospective study, so that the evaluations of the subjects must be systematically understood and controlled. Suggested perspectives for future investigations are longitudinal studies monitoring patients submitted to bone graft surgeries in order to perform a qualitative analysis of these individuals when facing these procedures.

\section{Conclusions}

Within the limitations of the current study, we may conclude that some clinical variables related to surgical treatment and to the limitation of activities/postoperative symptoms appear to be associated with the level of anxiety during surgical treatment for autogenous bone block graft. The perception of pain immediately after surgery did not predict the STAI-S after treatment, and there was an indication of causality regarding the STAI-T.

\section{References}

1. Esposito M, Grusovin MG, Felice P, Karatzopoulos G, Worthington HV, Coulthard P. Interventions for replacing missing teeth: horizontal and vertical bone augmentation techniques for dental implant treatment. Cochrane Database Syst Rev. 2009;7:CD003607.

2. Voss JO, Dieke T, Doll C, Sachse C, Nelson K, Raguse JD, et al. Retrospective long-term analysis of bone level changes after horizontal alveolar crest reconstruction with autologous bone grafts harvested from the posterior region of the mandible. J Periodontal Implant Sci. 2016;46:72-83.

3. Sakkas A, Wilde F, Heufelder M, Winter K, Schramm A. Autogenous bone grafts in oral implantology-is it still a "gold standard"? A consecutive review of 279 patients with 456 clinical procedures. Int J Implant Dent. 2017;3:23.

4. Khoury F, Hanser T. Mandibular bone block harvesting from the retromolar region: a 10-year prospective clinical study. Int J Oral Maxillofac Implants. 2015;30:688-97.

5. Hashem AA, Claffey NM, O'Connell B. Pain and anxiety following the placement of dental implants. Int J Oral Maxillofac Implants. 2006;21:943-50.

6. Kim S, Lee YJ, Lee S, Moon HS, Chung MK. Assessment of pain and anxiety following surgical placement of dental implants. Int J Oral Maxillofac Implants. 2013;28:531-5.

7. Weisensee W, Scheer M, Müller L, Rothamel D, Kistler F, Bayer $\mathrm{G}$, et al. Impact of anxiety parameters on prospective and experienced pain intensity in implant surgery. Implant Dent. 2012;21:502-6.

8. Goiato MC, Santiago Junior JF, Pellizzer EP, Moreno A, Villa LM, Dekon SF, et al. Systemic Trans- and postoperative evaluations of patients undergoing dental implant surgery. Clinics (Sao Paulo). 2016;71:156-62.

9. van Wijk AJ, de Jongh A, Lindeboom JA. Anxiety sensitivity as a predictor of anxiety and pain related to third molar removal. J Oral Maxillofac Surg. 2010;68:2723-9.

10. Cordaro L, Torsello F, Miuccio MT, di Torresanto VM, Eliopou- los D. Mandibular bone harvesting for alveolar reconstruction and implant placement: subjective and objective cross- sectional evaluation of donor and recipient site up to 4 years. Clin Oral Implants Res. 2011;22:1320-6.

11. Torres-Lagares D, Recio-Lora C, Castillo-Dalí G, Ruiz-de-LeónHernández G, Hita-Iglesias P, Serrera-Figallo MA, et al. Influence of state anxiety and trate anxiety in postoperative in oral surgery. Med Oral Patol Oral Cir Bucal. 2014;19:403-8.

12. Gaudry E, Vagg P, Spielberger CD. Validation of the State-Trait Distinction in Anxiety Research. Multivariate Behav Res. 1975;10:331-41.

13. Lago-Méndez L, Diniz-Freitas M, Senra-Rivera C, Seoane-Pesqueira G, Gándara-Rey JM, García-García A, et al. Postoperative recovery after removal of a lower third molar: role of trait and dental anxiety. Oral Surg Oral Med Oral Pathol Oral Radiol Endod. 2009;108:855-60.

14. Gorenstein C, Andrade L. Validation of a Portuguese version of the Beck Depression Inventory and the State-Trait Anxiety Inventory in Brazilian subjects. Braz J Med Biol Res. 1996;29:453-7.

15. Barker BM, Barker HR Jr, Wadsworth AP Jr. Factor analysis of the items of the State-Trait Anxiety Inventory. J Clin Psychol. 1977;33:450-5.

16. Diedenhofen B, Musch J. cocor: a comprehensive solution for the statistical comparison of correlations. PLoS One. 2015;10:e121945. 17. Camps-Font O, Burgueño-Barris G, Figueiredo R, Jung RE, Gay-Escoda C, Valmaseda-Castellón E. Interventions for dental implant placement in atrophic edentulous mandibles: vertical bone augmentation and alternative treatments. A meta-analysis of randomized clinical trials. J Periodontol. 2016;87:1444-57.

18. Eli I, Schwartz-Arad D, Baht R, Ben-Tuvim H. Effect of anxiety on the experience of pain in implant insertion. Clin Oral Implants Res. 2003;14:115-8.

19. Muglali M, Komerik N. Factors related to patients' anxiety before and after oral surgery. J Oral Maxillofac Surg. 2008;66:870-7.

20. Settineri S, Tati F, Fanara G. Gender differences in dental anxiety: is the chair position important? J Contemp Dent Pract. 2005;6:115-22. 21. Jeddy N, Nithya S, Radhika T, Jeddy N. Dental anxiety and influencing factors: A cross-sectional questionnaire-based survey. Indian J Dent Res. 2018;29:10-5.

22. Locker D. Psychosocial consequences of dental fear and anxiety. Community Dent Oral Epidemiol. 2003;31:144-51.

23. Ahmadi M, Kiakojori A, Moudi S. Association of anxiety with pain perception following periodontal flap surgery. J Int Soc Prev Community Dent. 2018;8:28-33.

\section{Acknowledgements}

The authors thank the Coordination for the Improvement of Higher Education Personnel (CAPES). JAAA is the recipient of scholarship. Mrs. E. Greene provided English editing of the manuscript.

\section{Conflicts of Interest}

The authors do not have any conflicts of interest. 